\title{
Transitivity and the patterns of adult preferences
}

\author{
H. BRADBURY and T. M. NELSON* \\ University of Alberta, Edmonton, Alberta, Canada
}

Eighty-four adults gave preference responses for exhaustive paired comparisons of three colors. An analysis is made of the patterns of response possible for the sequence of three choices involved, and the distribution of Ss' preferences over the available patterns is discussed. An explanation is offered for the patterned bias of adult preferences which relates choice context and the contingencies of choices made in sequence. Results are discussed in terms of transitivity and cognitive complexity.

A number of studies, notably Coombs (1958) and Tversky (1969), have been concerned with the intransitivity of sequences of preferences and the conditions that contribute to such inconsistency without making clear the relation of intransitive choice patterns to all other patterns possible. One developmental study (Smedslund, 1960) even carries the phrase "transitivity of preference patterns" in its title without ever specifying what these patterns are. This paper will examine the structure of one paired comparison choice situation and will discuss the distribution of a sample of adult preferences over the possible patterns of preference.

The transitive relation requires the choice $y<z$ following choices $x>y, x<z{ }^{1}$ This provides an ordinal relation of three items in such a way that the relation is implied in the first two choices and confirmed in the third. As in this example and in the Smedslund (1960) study of patterns of preference, the transitive problem can be confined to three choices which exhaust the paired comparisons of three items (i.e., first $x$ and $y$ presented and a choice made, then $\mathrm{x}$ and $\mathrm{z}$ presented and a choice made, then $y$ and $z$ presented and a choice made). In such a situation, there are eight possible patterns of preference, as shown in Table 1.

Two, or one-fourth, of the possible patterns of preference in the illustrated situation are intransitive. One observation that should be made is that intransitives are of different forms, initially distinguishable by the response in the first choice to the item which is to reappear in the second choice. In the illustrated case (i.e., $x y, x z, y z$ ), item $x$ is common to the first two pairs. One may provide an intransitive choice sequence either by selecting $\mathrm{x}$ in the first choice, rejecting $\mathrm{x}$ in the second choice, then in the third choice selecting the item not selected in the first choice (i.e., Form 7) or by rejecting $x$ in the first choice, selecting $x$ in the second choice, then in the third choice selecting the item not

*This research was partially supported by a grant awarded by the Division of Mental Health, Department of Health, Government of the Province of Alberta. selected in the second choice (i.e., Form 8). Both of these patterns and their transitive counterparts (i.e., Forms 5 and 6) have in common a shifting second choice. A shift is defined here as a second-choice change in the direction of response to the item common to the first two pairs of the three-pair choice sequence (i.e., either $x>y$ then $x<z$ or $x<y$ then $x>z$ ). It is only after a shifting second-choice response that an intransitive third-choice response can occur. One-half of the eight patterns of response contain a shift. The four patterns that do not contain a shift are considered nontransitive, since they are indeterminant so far as transitivity is concerned. Two of the shifting patterns are transitive; two are intransitive.

This breakdown of the patterns of preference responses shows that the $\mathrm{S}$ may avoid an intransitive response sequence either by shifting and then providing the transitive choice or by not shifting. Different contingencies obtain at each step in the three-choice sequence. Over a large sample of Ss, we should expect the two items in the first choice (i.e., $x$ and $y$ ) to be selected with roughly equal frequency, so long as the items used were not unequal in general attractiveness. In the second choice, where the shift becomes possible, we should once again logically expect the two items compared to be selected about equally frequently by a substantial sample of Ss. Because a person prefers item $\mathrm{x}$ to item y does not imply that he will prefer this same item $x$ to some other item $z$ or that, if he prefers item $y$ to item $\mathrm{x}$, he will also prefer some other item $\mathrm{z}$ to $\mathrm{x}$. However, once the first two choices have been made,

Table 1

The Eight Sets of Possible Preference Responses to a Sequence of All Paired Comparisons of Three Items, With Indication of the Transitivity (t), Nontransitivity (nt), or Intransitivity (c) of the Sets of Responses*

\begin{tabular}{llll}
\hline \multicolumn{1}{c}{1} & \multicolumn{1}{c}{3} & \multicolumn{1}{c}{3} & \multicolumn{1}{c}{4} \\
\hline$x>y$ & $x<y$ & $x>y$ & $x<y$ \\
$x>z$ & $x<z$ & $x>z$ & $x<z$ \\
$y>z$ & $y<z$ & $y<z$ & $y>z$ \\
nt & nt & nt & nt \\
No Shift & No Shift & No Shift & No Shift \\
\hline \multicolumn{1}{c}{5} & 6 & 7 & 8 \\
\hline$x>y$ & $x<y$ & $x>y$ & $x<y$ \\
$x<z$ & $x>z$ & $x<z$ & $x>z$ \\
$y<z$ & $y>z$ & $y>z$ & $y<z$ \\
$t$ & $t$ & $c$ & $c$ \\
Shift & Shift & Shift & Shift \\
\hline
\end{tabular}

*Shifting or nonshifting in each response form is also indicated. Shifting is responding differently in the second choice to the color common to the first two pairs of the three-pair choice sequence $(x>y, x<z$ or $x<y, x>z)$. 
Table 2

Patterns of Preference Responses by 84 Adults for All Paired Comparisons of Three Colors*

\begin{tabular}{|c|c|c|c|c|c|c|c|c|c|c|}
\hline \multirow[b]{2}{*}{ Colors } & & & \multicolumn{8}{|c|}{ Patterns of Response } \\
\hline & & & 1 & 2 & 3 & 4 & 5 & 6 & 7 & 8 \\
\hline 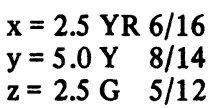 & $\begin{array}{l}\text { Frequency } \\
N=44\end{array}$ & $\begin{array}{l}\text { Males } \\
\text { Females }\end{array}$ & $\begin{array}{l}4 \\
2\end{array}$ & $\begin{array}{l}6 \\
5\end{array}$ & $\begin{array}{l}3 \\
2\end{array}$ & 3 & $\begin{array}{l}7 \\
4\end{array}$ & $\begin{array}{l}2 \\
2\end{array}$ & $\begin{array}{l}2 \\
1\end{array}$ & 1 \\
\hline 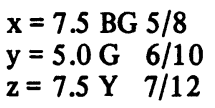 & $\begin{array}{l}\text { Frequency } \\
N=40\end{array}$ & $\begin{array}{l}\text { Males } \\
\text { Females }\end{array}$ & $\begin{array}{l}3 \\
5\end{array}$ & $\begin{array}{l}6 \\
4\end{array}$ & & $\begin{array}{l}4 \\
5\end{array}$ & $\begin{array}{l}3 \\
1\end{array}$ & $\begin{array}{l}1 \\
3\end{array}$ & $\begin{array}{l}1 \\
1\end{array}$ & $\begin{array}{l}2 \\
1\end{array}$ \\
\hline \multirow[t]{2}{*}{ Totals } & Frequency & $\begin{array}{l}\text { Males } \\
\text { Females }\end{array}$ & $\begin{array}{l}7 \\
7\end{array}$ & $\begin{array}{r}12 \\
9\end{array}$ & $\begin{array}{l}3 \\
2\end{array}$ & $\begin{array}{l}4 \\
8\end{array}$ & $\begin{array}{r}10 \\
5\end{array}$ & $\begin{array}{l}3 \\
5\end{array}$ & $\begin{array}{l}3 \\
2\end{array}$ & $\begin{array}{l}2 \\
2\end{array}$ \\
\hline & $N=84$ & $\begin{array}{l}\text { All Ss } \\
\text { Percent }\end{array}$ & $\begin{array}{l}14 \\
17\end{array}$ & $\begin{array}{l}21 \\
25\end{array}$ & $\begin{array}{l}5 \\
6\end{array}$ & $\begin{array}{l}12 \\
14\end{array}$ & $\begin{array}{l}15 \\
18\end{array}$ & $\begin{array}{r}8 \\
10\end{array}$ & $\begin{array}{l}5 \\
6\end{array}$ & $\begin{array}{l}4 \\
5\end{array}$ \\
\hline
\end{tabular}

*The Munsell specifications for the colors used are shown with the data for each condition. The colors in each pair were shown in succession. The first-second position of items in pairs was randomly determined. The patterns of response to which the table refers are the eight possible ways of responding to three pairs which exhaust the paired comparisons of three items.

constraints are established for some third choices, which is the crux of the transitive relation. In the third choice, $\mathrm{y}$ and $\mathrm{z}$ are compared. Logically we should expect $\mathrm{y}$ and $z$ to be selected about equally frequently after $x$ has been preferred to both of them, and we should expect the same results when both $y$ and $z$ have been preferred to $x$. But once $x$ ha been preferred to $y$ and $z$ has been preferred to $\mathrm{x}$, the rule would predict preference for $\mathrm{z}$ over $\mathrm{y}$. Similarly, preference for $\mathrm{y}$ over $\mathrm{x}$ and $\mathrm{x}$ over $\mathrm{z}$ implies preference of $\mathbf{y}$ over $\mathbf{z}$.

\section{METHOD}

Subjects

Eighty-four undergraduate university students enrolled in introductory psychology classes served as Ss. All were tested for color blindness using H-R-R pseudoisochromatic plates.

\section{Materials and Procedure}

Two sets of colors were selected on the basis of their being well named or poorly named by English speaking adults (Chapanis, 1965). Following are the Munsell specifications for colors considered well named: 2.5 YR $6 / 16$ (orange), 5.0 Y 8/14 (yellow), 2.5 G 5/12 (green); and not well named: 7.5 BG 5/8, $5.0 \mathrm{G} \mathrm{6/10,7.5} \mathrm{Y} \mathrm{7/12.} \mathrm{These} \mathrm{are} \mathrm{all} \mathrm{highly} \mathrm{saturated} \mathrm{hues} \mathrm{of}$ medium lightness. The color samples used were $6 \times 10$ in. Munsell papers with glossy finish. The papers were mounted on a sliding tray of a neutral gray screen, with a single window facing the $S$ so as to allow successive exposure of the items in pairs. The colors in each pair were each shown for $5 \mathrm{sec}$ in immediate succession, with a 10-sec interval between each pair during which preferences were marked on response sheets provided.

\section{RESULTS}

Table 2 shows the distributions of Ss' responses according to sex and the color set to which preferences were given. Intransitive preference patterns occurred less frequently than would have been expected by chance $\left(\chi^{2}=9.15\right.$, df $\left.=1, p<.01\right)$. This indicates that the constraints of logical relations are reflected in personal preferences, but the level of intransitivity obtained (i.e., $11 \%$ ) is substantially in excess of the requirements of the logical rule. The two sets of colors do not differ in proportion of intransitive preference responses.

The data support the 50-50 expectation for first choices (i.e., color $x$ was preferred to color $y$ in the first pair about half the time). The second choice conformed to expectations when $x$ had been chosen in the first pair but not when $y$ had been chosen in the first pair. After $x$ had been chosen in the first pair (i.e., 46\%), the same color was chosen in the second pair about half the time (i.e., 49\%). However, after $y$ had been chosen over $x$ in the first pair (54\%), $\mathrm{x}$ was infrequently chosen in the second pair (27\%).

In the third choices, $\mathrm{z}$ was more frequently chosen (64\%) than y (36\%) after y had been preferred in the first pair and $z$ had been preferred in the second pair. The transitive y was more frequently chosen (67\%) over the intransitive $\mathrm{z}(33 \%)$ after $\mathrm{y}$ had been preferred in the first pair and $x$ had been preferred in the second pair. Similarly, the transitive $\mathrm{z}$ was more frequently chosen (75\%) than $y(25 \%)$ after $\mathrm{x}$ had been preferred in the first pair and $z$ had been preferred in the second pair. But y was more frequently chosen (75\%) than $z$ (26\%) after $x$ had been preferred in both the first and second pairs. These results for choices in sequence are shown in Table 3.

\section{DISCUSSION}

The preference responses conform to logical expectations and depart from them in peculiarly patterned ways. It was observed that if a person chooses $x$ over $y$ and then is asked to choose between $x$ and $z$, we should not be able to predict his preference better than chance. The data support this. However, an analogous relation is not supported. The equal preference for $\mathbf{x}$ and $z$ in the second choice is not supported when $y$ has been preferred to $x$ in the first choice. If a $S$ has not preferred $x$ in the first choice, it is relatively unlikely that he will prefer it in the second choice. Shifting is unlikely if the item not chosen in the first pair reappears in the second pair (i.e., if it is rejected once, it is likely that it will be rejected again).

Third choices are similarly perplexing. In the two choices in which transitivity is at issue, the data are consistent with realistic as opposed to purely logical expectations. The data show a predominance of transitive choices with a notable level of intransitive choices. Explanations for the occurrence of the 11\% of responses which were intransitive would differ, but the very concept of stochastic transitivity supports an expectation of some such level of inconsistency in a population of choices. So the overall .71 level of third-choice transitivity is consistent with general findings for such decisions, and the two different 
transitive forms do not differ greatly in this respect (i.e., .75 transitive for one and .67 transitive for the other).

Both the transitive bias and the instances of intransitivity in choices involving possible intransitivity are not surprising. However, results for the other two third choices (the nontransitive choices) are just as biased as those involving transitive constraints. This is a bit surprising. Once Ss have preferred $x$ to both $y$ and $z$, they most frequently (75\%) preferred $y$ to $z$. In a corresponding though reversed fashion, once they have preferred both $y$ and $z$ to $x$, they most frequently (i.e., 64\%) preferred $z$ to $y$. All third choices are between $y$ and $z$. All of these choices (there are four of them) are strongly biased in favor of one or the other of the two items, and the biases are so coordinated that the two items ( $y$ and $z$ ) are selected equally frequently overall.

The problem is to account for the biases toward nontransitive choices. Considering first the second-choice options, we can see a relation between shifting, novelty aspects of the choice context, and the logical consequences of responding to novelty. These novelty influences must include both a tendency to choose an object that is new and a tendency to respond to available objects in a new way. In the choice situation illustrated in this study, these two factors sometimes work together and sometimes work at odds. The clearest instance of convergence of these alleged novelty influences is seen in the second-choice comparison of $x$ and $z$ after selection of $x$ in the first choice. Here selection of $z$ is both a response for the new stimulus and a new response relative to $x$ (i.e., $x$ was selected in the first choice, it is rejected in the second choice). The data indicate no novelty bias for $z$ in this choice. However, to select $\mathrm{z}$ in this case is to shift and allow the possibility of an intransitive third choice. There is a considerable logical advantage in, so to speak, staying with the first choice. The cognitive relations are simplified by selecting $\mathrm{x}$ in the second choice after selection of $\mathrm{x}$ in the first choice.

In the other second-choice situation (i.e., comparison of $x$ and $z$ after selection of $y$ in the first choice), the alleged novelty influences are seen in a different combination. In this case, selection of $z$ is a response for the new stimulus; however, selection of $x$ is a new response ( $x$ was not preferred in the first choice, it is preferred in the second choice). The distinguishing features of this choice situation in contrast to the other second choice already discussed are: (1) new stimulus and new response features are in conflict, and (2) the new stimulus selection does not produce a shifting response pattern. In this situation, response is strongly biased in favor of the new stimulus (73\%). It is suggested that one form of shift (i.e., $x>y, x<z$ ) is more common than the other (i.e., $x<y, x>z$ ) because of opposite new stimulus features in the two patterns. Both shifts are new response patterns by definition, but one of them contains new stimulus influences in favor of a nonshifting preference. This view of the data suggests that the Ss' second choice in the three-choice sequence is under conflicting pressures (1) to choose a new stimulus and to respond in a new way to available stimuli and (2) to reduce the logical difficulty or cognitive complexity of relations created by the act of choosing.

The two sets of nontransitive third choices illustrate the continuation of patterns of response established in the second choices. The $\mathrm{S}$ comes to a necessarily nontransitive third choice by having just given a nonshifting response to the second choice (i.e., if he chose $x$ in the first pair, he chose it again; if he did not choose $\mathrm{x}$ in the first pair, he did not choose it again). The biased third choices are continuations of these response inclinations. If the crucial aspect of nonshifting may be extended to the third choices, we can see that those Ss who tended not to shift in their second choices also tended not to shift in their third choices. Preference for $y$ over $z$ in the third choice after preference for $x$ over $\mathrm{z}$ in the second choice is a nonshifting response form relative to $z$. Also, preference for $z$ over $y$ in the third choice after preference for $\mathrm{z}$ over $\mathrm{x}$ in the second choice is a nonshifting response form relative to $z$. All of the Ss who gave these responses had established nonshifting forms of response relative to $x$ in their second choices. It might be noticed that the first of
Table 3

The Sequences of Choices Possible in Exhaustive Paired Comparisons of Three Colors (i.e., First Pair, $x$ and $y$; Second Pair, $x$ and $z$; Third Pair, $y$ and $z$ ) and the Proportions of Preferences at Each Option by 84 Adults

$\begin{array}{lcc}\text { Choice in } & \text { Choice in } & \text { Choice in } \\ \text { First Pair Second Pair } & \text { Third Pair }\end{array}$

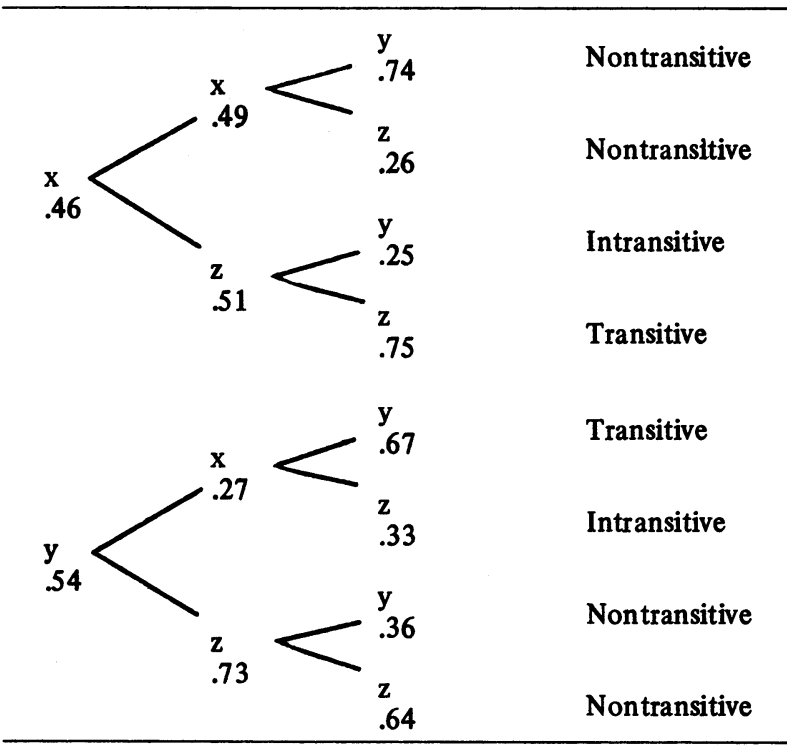

these two response forms $(x>y, x>z, y>z)$ was somewhat more biased in its third choice than was the other $(x<y, x<z$, $y<z)$, the relation being $74 \%$ to $64 \%$. This should be expected since bias for $y$ in this form is also a bias in favor of the more novel stimulus; $y$ is newer in the sequence than $z$ in this third-choice comparison. But the most salient organizational feature of these response forms is the tendency toward nonshifting by some Ss.

The nonshifting mode of response shown completely by $42 \%$ of the Ss (i.e., Forms 1 and 2 in Table 1) represents the most simplified and cognitively expeditious organization of the relations available in the three-choice sequence (i.e., $x>y$, $x>z, y>z$ is a less complex and less problematic relation than $\mathrm{x}>\mathrm{y}, \mathrm{x}<\mathrm{z}, \mathrm{y}<\mathrm{z}$ ).

The important aspect of the issue of cognitive complexity for choice context is that the $S$ creates the relations facing him by the choices he makes. The act of choice early in a sequence of comparisons of the sort illustrated here establishes the contingencies facing the chooser later. Adult preferences are biased in favor of certain patterns explainable on the basis of these contingencies. This is why Patterns 1 and 2 in Table 1 are so common and Patterns 7 and 8 are so uncommon.

\section{REFERENCES}

Chapanis, A. Color names for color space. American Scientist. $1965,53,327-346$.

Coombs, C. H. On the use of inconsistency of preferences in psychological measurement. Journal of Experimental Psychology, 1958, 55, 1-7.

Smedslund, J. Transitivity of preference patterns as seen by preschool children. Scandinavian Journal of Psychology, $1960,1,49-54$.

Tversky, $A$. Intransitivity of preferences. Psychological Review, $1969,76,31-48$.

\section{NOTE}

1. The symbols $>$ and $<$ are used to indicate "is preferred to." $x>y$ indicates $x$ is preferred to $y$.

(Received for publication February 21, 1973.) 